\title{
Sildenafil, a cyclic GMP phosphodiesterase inhibitor, induces microglial modulation after focal ischemia in the neonatal mouse brain
}

Raffaella Moretti ${ }^{1,2}$, Pierre-Louis Leger ${ }^{1,3}$, Valérie C. Besson ${ }^{1,4}$, Zsolt Csaba ${ }^{1}$, Julien Pansiot ${ }^{1}$, Lorena Di Criscio $^{1}$, Andrea Gentilii, Luigi Titomanlio ${ }^{1,7}$, Philippe Bonnin ${ }^{5,6}$, Olivier Baud ${ }^{1,8}$ and Christiane Charriaut-Marlangue ${ }^{1,9^{*}}$

\begin{abstract}
Background: Perinatal ischemic stroke is the most frequent form of cerebral infarction in neonates; however, evidence-based treatments are currently lacking. We have previously demonstrated a beneficial effect of sildenafil citrate, a PDE-5 inhibitor, on stroke lesion size in neonatal rat pups. The present study investigated the effects of sildenafil in a neonatal mouse stroke model on (1) hemodynamic changes and (2) regulation of astrocyte/microgliamediated neuroinflammation.

Methods: Ischemia was induced in C57BI/6 mice on postnatal (P) day 9 by permanent middle cerebral artery occlusion (pMCAo), and followed by either PBS or sildenafil intraperitoneal (i.p.) injections. Blood flow (BF) velocities were measured by ultrasound imaging with sequential Doppler recordings and laser speckle contrast imaging. Animals were euthanized, and brain tissues were obtained at $72 \mathrm{~h}$ or 8 days after pMCAo. Expression of M1- and M2-like microglia/macrophage markers were analyzed.

Results: Although sildenafil (10 mg/kg) treatment potently increased cGMP concentrations, it did not influence early collateral recruitment nor did it reduce mean infarct volumes $72 \mathrm{~h}$ after pMCAo. Nevertheless, it provided a significant dose-dependent reduction of mean lesion extent 8 days after pMCAo. Suggesting a mechanism involving modulation of the inflammatory response, sildenafil significantly decreased microglial density at $72 \mathrm{~h}$ and 8 days after pMCAo. Gene expression profiles indicated that sildenafil treatment also modulates M1- (ptgs2, CD32 and CD86) and M2-like (CD206, Arg-1 and Lgals3) microglia/macrophages in the late phase after pMCAo. Accordingly, the number of COX-2 $2^{+}$microglia/macrophages significantly increased in the penumbra at $72 \mathrm{~h}$ after pMCAo but was significantly decreased 8 days after ischemia in sildenafil-treated animals.

Conclusions: Our findings argue that anti-inflammatory effects of sildenafil may provide protection against lesion extension in the late phase after pMCAo in neonatal mice. We propose that sildenafil treatment could represent a potential strategy for neonatal ischemic stroke treatment/recovery.
\end{abstract}

Keywords: Neonatal mice, Focal ischemia, Microglia, M2 microglia, Microcirculation

\footnotetext{
* Correspondence: christiane.marlangue@gmail.com

Olivier Baud and Christiane Charriaut-Marlangue share senior authorship.

${ }^{1}$ University Paris Diderot, Sorbonne Paris Cité, INSERM, UMR 1141, 75019

Paris, France

9INSERM UMR 1141, Hopital Robert Debré, 48 bd Serurier, 75019 Paris, France

Full list of author information is available at the end of the article
} 


\section{Background}

Perinatal/neonatal arterial stroke is a cerebrovascular event occurring around the time of birth, with pathological or radiological evidence of focal arterial infarction mainly affecting the middle cerebral arterial territory, with an incidence of $1 / 2800$ to $1 / 5000$ live births. There is currently no evidence-based treatment for neonates with stroke [1].

Decreased regional cerebral blood flow $(\mathrm{CBF})$ is the principal factor determining the topography of tissue injury after cerebral ischemia and/or hypoxia-ischemia (HI) in the mature and immature rodents [2]. Partially maintained blood flow in the penumbral tissue after stroke is primarily due to the recruitment of collateral circulation in the brain. Collateral recruitment involves nitric oxide (NO)-dependent vasodilatation, and the effects of endogenous $\mathrm{NO}$ may be enhanced by preventing cGMP degradation by phosphodiesterases (PDEs). In particular, sildenafil and tadalafil, two potent selective PDE-5 inhibitors, prolong the action of cGMP in multiple vascular territories [3]. Interestingly, sildenafil treatment, by increasing $\mathrm{CBF}$, reduced HI damage in the P7 rat brain [4]. Sildenafil has been reported to promote neurorestoration in rat models of stroke as measured by neurogenesis, synaptogenesis, and angiogenesis [5] and to reduce proliferation of astrocytes and microglia in chronic inflammatory diseases [6].

While microglia are considered as conductors of the inflammatory response in the central nervous system (CNS), their contribution to the progression of ischemic stroke remains debated [7]. Microglia/macrophages may transition between inflammatory and healing phenotypes in response to specific activation cues (review in [8]). While the M1 or classic phenotype is neuroinflammatory and neurotoxic, the M2 phenotype supports the recovery or neural tissue and is characterized by the production of anti-inflammatory mediators and neurotrophic factors [9]. Although the action of sildenafil on neuroinflammation is not fully understood, the cGMPPDE inhibitor zaprinast was shown to significantly reduce the inflammatory response of astrocytes and microglia/macrophages in adult rats submitted to focal traumatic brain injury [10].

Revealing molecular mechanisms and pharmacological treatments that promote the transition of microglia/ macrophages towards a phenotype that facilitates the repair of the neonatal brain after ischemic damage is of therapeutic interest. The goal of this study is to examine if sildenafil citrate (1) mediates $\mathrm{CBF}$ and reduces lesion damage and (2) modifies the modulation of microglia/ macrophage between M1 and M2 phenotypes in P9 mice subjected to ischemia after permanent middle cerebral artery occlusion (pMCAo).

\section{Methods}

Ethics statement

All experiments were carried out (license A75-19-01, French Department of Agriculture) in accordance with the European Committee's Council Directive and performed to comply with the ethical guidelines of the Robert Debre Hospital Research Council Review Board and have been approved by the local ethic committee (Paris7, France).

\section{Ischemia}

pMCAo was carried out under isoflurane anesthesia in $30 \% \mathrm{O}_{2}$ and $70 \% \mathrm{~N}_{2} \mathrm{O}$ in $\mathrm{P} 9 \mathrm{C} 57 \mathrm{Bl} / 6$ mouse (Janvier, Le Genest-St. Isle, France; $4.6 \pm 0.6 \mathrm{~g}, n=148)$. Pups were sacrificed at $72 \mathrm{~h}$ and/or 8 days after pMCAo. Two investigators, both blinded to the treatment group, determined the size of the lesion in each animal [11].

\section{Drug treatment}

In the first set of experiments, animals were randomly divided into five groups and treated with either PBS or a single dose of sildenafil citrate $(0.5,2.5,10$, and $15 \mathrm{mg} / \mathrm{kg}$; Pfizer, France), given intraperitoneally (i.p.) 5 min after pMCAo. In the second set of experiments, animals were randomly divided into three groups and treated with either PBS or a single dose of sildenafil citrate (0.5 and $10 \mathrm{mg} / \mathrm{kg}$, i.p.) $5 \mathrm{~min}$ after pMCAo (see Additional file 1: Figure S1 for an outline of the experimental procedure).

\section{cGMP measurement}

Competitive enzyme immunoassay (Cayman Chemical Company, Ann Arbor, MI, USA) was used to quantify cGMP in the forebrain, according to the manufacturer's instructions. Whole brains at P9 were harvested 1 and $3 \mathrm{~h}$ after the administration of sildenafil ( 0.5 and/or $10 \mathrm{mg} / \mathrm{kg}$ ) and immediately frozen at $-80{ }^{\circ} \mathrm{C}$ until measurements were performed.

\section{Ultrasonographic brain imaging}

Thermoregulated mice ( $n=6$ per group) were subjected to ultrasound measurements under inhaled isoflurane anesthesia ( $0.8 \%$ in air via a facemask) using an echograph (ACUSON S3000, Siemens, Erlangen, Germany) equipped with a $14.5-\mathrm{MHz}$ linear transducer (14L5 SP) [12]. Heart rate and time-average mean blood flow velocities (mBFVs) were measured in both intracranial carotid arteries (ICA) and the basilar trunk (BT) at baseline and $1 \mathrm{~h}$ after pMCAo and PBS and sildenafil (10 mg/kg) treatment.

\section{Laser speckle contrast (LSC) imaging}

Imaging was carried out using a full-field laser perfusion imager (FLPI2, Moor Instruments Ltd., Axminster, UK) 
[12]. Briefly, thermoregulated and anesthetized (with $1 \%$ isoflurane inhalation in air via a facemask) mouse pups ( $n=6$ per group) were placed in lateral decubitus and the skin incised to provide access to the skull. Vegetable oil was applied to avoid surface dryness. Speckle images $(760 \times 568$ pixels $)$ were collected at $0.25 \mathrm{~Hz}$ (4 ms exposure time) and recorded at baseline, after MCA electrocoagulation and 5, 15, 60, and $90 \mathrm{~min}$ after treatment. Blood fluxes were measured in three cortical regions of interest (ROI, $50 \times 50$ pixels), ROI 1 present in the core of the infarct and ROI 2 and ROI 3 in the proximal and distal penumbra, respectively. Fluxes in the ROIs were expressed in arbitrary units using a 16-color palette.

\section{Real-time quantitative reverse transcriptase polymerase chain reaction ( $\mathrm{QRT}-\mathrm{PCR}$ )}

Animals were sacrificed at $72 \mathrm{~h}$ and 8 days after pMCAo ( $n=8$ per group). The harvested tissues correspond to the parietal cortex, including the ischemic core (pale zone) and penumbra at $72 \mathrm{~h}$, and the penumbra at 8 days as the pale zone being a cavity. Total RNA was extracted using RNeasy lipid tissue mini kit (Qiagen, Courtaboeuf, France). RNA purity and quality were assessed by spectrophotometry with the NanoDrop ${ }^{\mathrm{TM}}$ apparatus (Thermo Scientific, Wilmington, DE, USA). Total RNA (1-2 $\mu \mathrm{g})$ was subjected to reverse transcription using the iScript ${ }^{\mathrm{TM}}$ cDNA synthesis kit (Bio-Rad, Marnes-la-Coquette, France). RT-qPCR was performed in duplicates for each sample using SYBR Green Supermix (Bio-Rad) for $40 \mathrm{cy}$ cles with a 2-step program $\left(5 \mathrm{~s}\right.$ of denaturation at $96{ }^{\circ} \mathrm{C}$ and $10 \mathrm{~s}$ of annealing at $\left.60{ }^{\circ} \mathrm{C}\right)$. Amplification specificity was assessed by melting curve analysis. Primers were designed using Primer3 software. Sequences and NCBI references are provided in Additional file 2: Table S1. Abundance of transcripts of interest was expressed relative to the expression of the reference gene, glyceraldehyde 3-phosphate dehydrogenase $(G A P D H)$. Analyses were performed with the Bio-Rad CFX Manager 2.1 software.

\section{Immunohistochemistry}

Coronal $16-\mu \mathrm{m}$ thick paraffin sections at the MCA and hippocampal level (corresponding to 3.27 and $4.83 \mathrm{~mm}$-atlas of the developing mouse brain at P6) were stained for mouse anti-GFAP (Sigma-Aldrich G3893; 1:500 dilution), goat anti-Iba1 (Abcam ab5076; 1:500), rabbit anti-Iba1 (Wako, 019-19741; 1:1000), goat anti-MRC1 (MMR/CD206 polyclonal Goat IgG, R\&D system, AF2535; 1:400), rabbit anti-COX-2 (Abcam; 1:200), goat anti-arginase-1 (Santa Cruz Biotechnology, Inc., sc-18354; 1:200), rabbit anti-Glut1 (54-KDa form, Millipore, Molsheim, France; 1:700), and tomato lectin (Vector Laboratories, 1:500). For immunofluorescence, secondary antibodies coupled with the green marker
Fluoroprobe S488 (Interchim, Montluçon, France) or the red fluorescent marker cyanine 3 (Jackson Immuno Research laboratories, West grove, PA) were used. For confocal imaging, sections were analyzed using a Leica TCS SP8 confocal scanning system (Leica Microsystems, Wetzlar, Germany) equipped with 405-nm diode, 488-nm argon, and 561-nm DPSS lasers. A series of optical sections separated by $0.3 \mu \mathrm{m}$ was collected using the $\times 63 \mathrm{HC}$ PL APO CS2 objective (numerical aperture 1.40). For each optical section, double- or triple-immunofluorescence images were acquired in sequential mode to avoid potential contamination by fluorescence emission overlap. The orthogonal sectioning and $3 \mathrm{D}$ viewing were produced by the Leica Application Suite X-software (Leica Microsystems). Images were equally adjusted for brightness and contrast, and composite illustrations were built in Adobe Photoshop CS3 (Adobe Systems, San Jose, CA).

\section{Cell quantification}

GFAP and Iba-1-positive cells were counted in a blind manner in the penumbra region on three coronal sections for animal at $72 \mathrm{~h}$ and 8 days after ischemia and in age-matched controls using a $\times 20$ objective. Iba-1-Arg-1 and Iba1-COX-2 double-positive cells were counted in the same regions with a $\times 20$ lens and then merged images were created with Fiji software (http://fiji.sc/Fiji).

\section{Statistical analysis}

Values are expressed as mean \pm SD (BF measures and lesion volume) and mean \pm SEM (RT-qPCR, immunohistochemistry, and immunofluorescence). BFV and CBF values measured in the different ROIs were compared using repeated measures ANOVA and a post hoc Newman-Keuls test to analyze differences between the two groups. One-way ANOVA followed by a Newman-Keuls post hoc test was used for the difference between two groups (RT-qPCR, immuno-labeled cells, and lesion volumes). Statistical analyses were performed using GraphPad PRISM version 5.0 (GraphPad Software, San Diego, CA).

\section{Results}

Setup of the animal model and effect of sildenafil on CBF and lesion size

In the first set of experiments (Additional file 1: Figure S1), animals were assessed by color-coded pulsed Doppler ultrasound imaging at baseline and after pMCAo. As compared to basal mBFV, pMCAo induced a significant transient decrease in mBFV in the left ICA and a consecutive significant increase in $\mathrm{mBFV}$ in the basilar trunk (BT) $15 \mathrm{~min}$ after pMCAo (Additional file 1: Figure S2A). One hour after pMCAo, no significant change in $\mathrm{MBFV}$ was observed in the three large arteries (data not shown). One hour after pMCAo and sildenafil administration $(10 \mathrm{mg} / \mathrm{kg}, n=8$ 
each) mBFVs were not modified in the three large arteries as compared to a single PBS administration (Fig. 1a). Heart rates in the sildenafil-treated animals $(395 \pm 67 \mathrm{bpm})$ were not significantly different from PBS-treated animals (428 \pm $66 \mathrm{bpm}$ ) at this time point. No difference was observed between the different doses of sildenafil on mBFVs measured in the large arteries (data not shown), except for the $15 \mathrm{mg} /$ $\mathrm{kg}$ dose (Additional file 1: Figure S2B).

Representative patterns and values of cortical BF using LSC imaging are shown in Fig. 1b, c. Immediately after pMCAo, BF dropped in the ROI 1 (core) to a mean value around $40-42 \%$ of basal $\mathrm{BF}$ in both groups of animals ( $n=6$ each) and was reduced further to $33-36 \%$ of basal at 90 min after pMCAo. A similar drop in BF was measured in the ROI 2 and in a less severe manner in the ROI 3. BF was further reduced at $90 \mathrm{~min}$ after pMCAo, in particular in ROI 3. As expected, sildenafil treatment $(10 \mathrm{mg} / \mathrm{kg})$ significantly increased the concentration of intracellular cGMP to $1.91 \pm 0.38$ and $1.87 \pm 0.31 \mathrm{pmol} / \mathrm{mL}$ at 1 and $3 \mathrm{~h}$ after administration, respectively, as compared to PBS-treated animals (0.08 $\pm 0.02 \mathrm{pmol} / \mathrm{mL}, p<0.001$ ) (Additional file 1: Figure S3). No statistical difference in cGMP concentration was found between the different doses used. Nevertheless, sildenafil treatment did not significantly modify the BF profile in the three distinct ROIs at any time point.

Mean cortical infarct volume at $72 \mathrm{~h}$ after pMCAo was $12.5 \pm 3.0 \%(n=14$, one animal died $)$ in PBS-treated animals. Sildenafil at the three lower doses tested $(0.5,2.5$, and $10 \mathrm{mg} / \mathrm{kg})$ did not change the mean infarct volume $(11.0 \pm$ $3.0 \%, n=7 ; 12.2 \pm 3.1 \%, n=8$; and $13.4 \pm 2.3 \%, n=7$, respectively, N.S.), whereas it increased both infarct volume $(21.6 \pm 4.6 \%, n=7, p<0.001$, Fig. $2 \mathrm{a})$ and mortality (3/10 animals died) at $15 \mathrm{mg} / \mathrm{kg}$, suggesting toxicity at high doses (Fig. 2a). Therefore, the $15-\mathrm{mg} / \mathrm{kg}$ dose was not further used in the following experiments. Eight days after

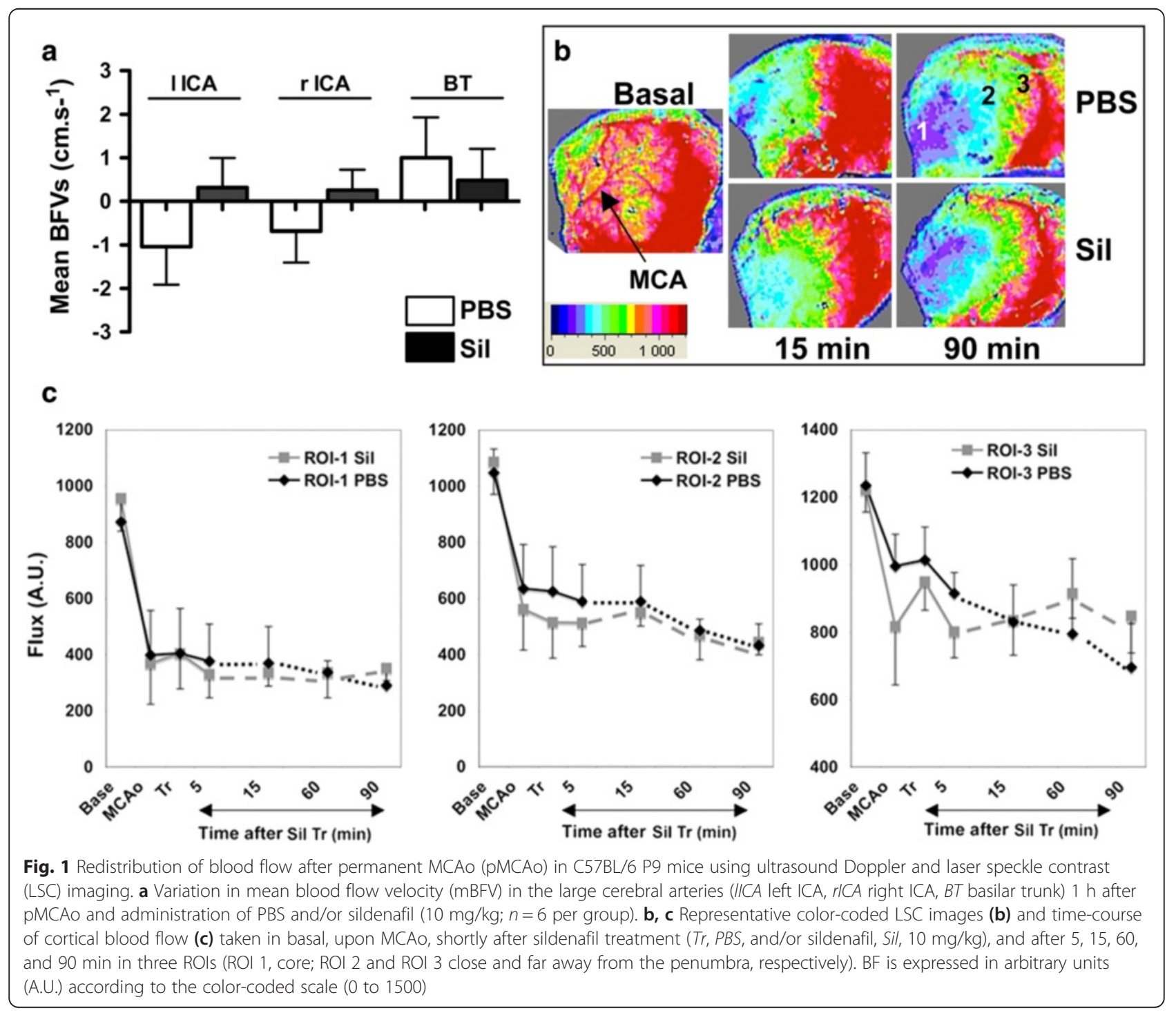



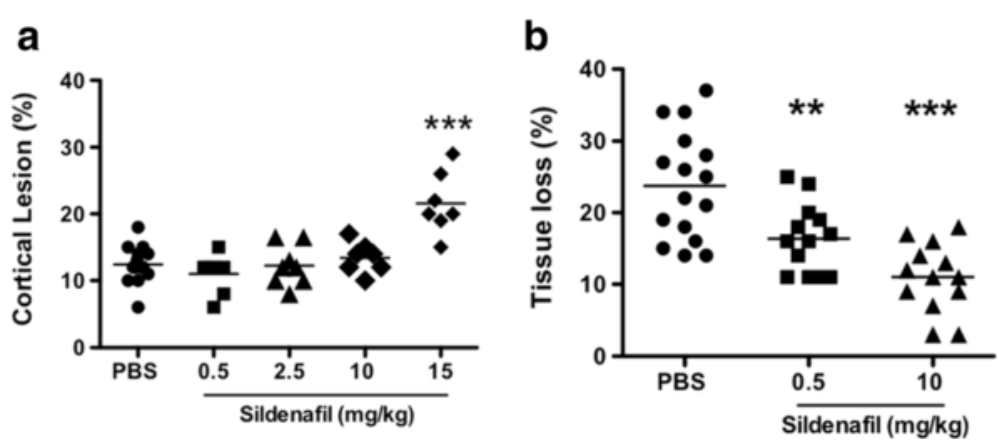

Fig. 2 Effect of sildenafil citrate on the cortical infarct volumes and tissue loss measured $72 \mathrm{~h}$ and 8 days after pMCAo, respectively. Sildenafil citrate was given after pMCAo by a single intraperitoneal injection either with PBS or $0.5,2.5,10$, and $15 \mathrm{mg} / \mathrm{kg}$ sildenafil. a Cortical lesion at $72 \mathrm{~h}$. b Tissue loss at 8 days. Horizontal bar represents the mean. Note that the $15 \mathrm{mg} / \mathrm{kg}$ dose is toxic by increasing the infarct volume. Each dot represents one animal. Data were assessed via an ANOVA, and when significant the results of the Newman-Keuls post-test are shown, ${ }^{* *} p<0.01$, *** $p<0.001$ sildenafil dose vs PBS
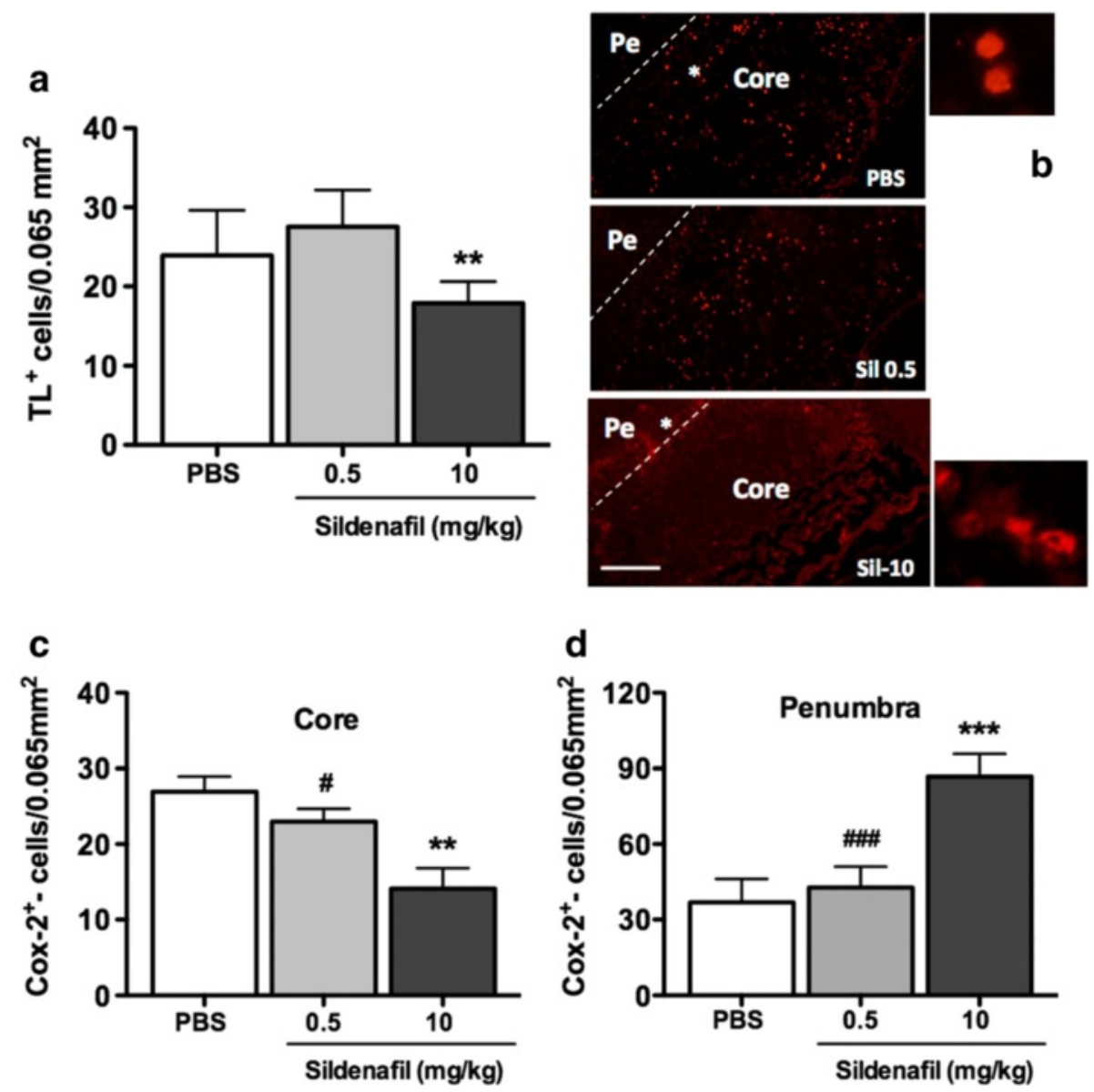

Fig. 3 Effect of sildenafil citrate on the density of microglia/macrophages cells and COX-2 expression at $72 \mathrm{~h}$ after pMCAo. a Quantitative analysis of tomato-lectin ( $T L, n=6-7$ per group) in PBS, Sil-0.5, and Sil-10 (mg/kg)-treated animals. $\mathbf{b}-\mathbf{d}$ Localization and quantification of COX-2 ${ }^{+}$cells in the core and/or the penumbra in PBS- and sildenafil-treated animals. ${ }^{* *} p<0.01,{ }^{* * *} p<0.001$, sildenafil vs PBS treatment. ${ }^{\# \#} p<0.01$, ${ }^{\# \# \#} p<0.001$, sildenafil 0.5 vs sildenafil 10. Scale bar represents $100 \mu \mathrm{m}$ (b) and $50 \mu \mathrm{m}$ (enlarged panels) 
pMCAo, lesion volume significantly increased up to 23.8 $\pm 7.5 \%(n=16, p<0.01$ vs $72 \mathrm{~h})$ in PBS-treated animals and became cystic. Sildenafil significantly counteracted this increase in a dose-dependent manner to $16.4 \pm 4.8 \%$ (sildenafil $0.5 \mathrm{mg} / \mathrm{kg} ; n=13 ; p<0.01$ ) and $11.0 \pm 4.8 \%$ (sildenafil $10 \mathrm{mg} / \mathrm{kg} ; n=13 ; p<0.001$ ) (Fig. 2b).

\section{Effect of sildenafil treatment on glial activation}

Reactive astrogliosis manifested by a significant increase in GFAP immunoreactivity was clearly observed around the lesion in PBS-treated animals at $72 \mathrm{~h}$ after pMCAo (Additional file 1: Figure S4 and Additional file 1: Figure S5A, B). Surprisingly, sildenafil significantly increased the prevalence of $\mathrm{GFAP}^{+}$ cells at $10 \mathrm{mg} / \mathrm{kg}$. Conversely, sildenafil significantly reduced the number of $\mathrm{GFAP}^{+}$cells 8 days after pMCAo as compared to PBS-treated animals. No significant difference was observed between PBS and $0.5 \mathrm{mg} / \mathrm{kg}$ sildenafil treatment at either time point (Additional file 1: Figure S4).

A high abundance of microglia/macrophages (stained by tomato lectin (TL)) was detected in the penumbra (including the white matter) at $72 \mathrm{~h}$. Sildenafil treatment significantly decreased the number of $\mathrm{TL}^{+}$-cells at 10 but not $0.5 \mathrm{mg} / \mathrm{kg}$ (Fig. 3a and Additional file 1: Figure S5C, D). At this time point, cells positive for the M1-like marker $\mathrm{COX}_{-} 2^{+}[13]$ were found in the ischemic core in PBStreated animals, whereas they were mostly observed in the

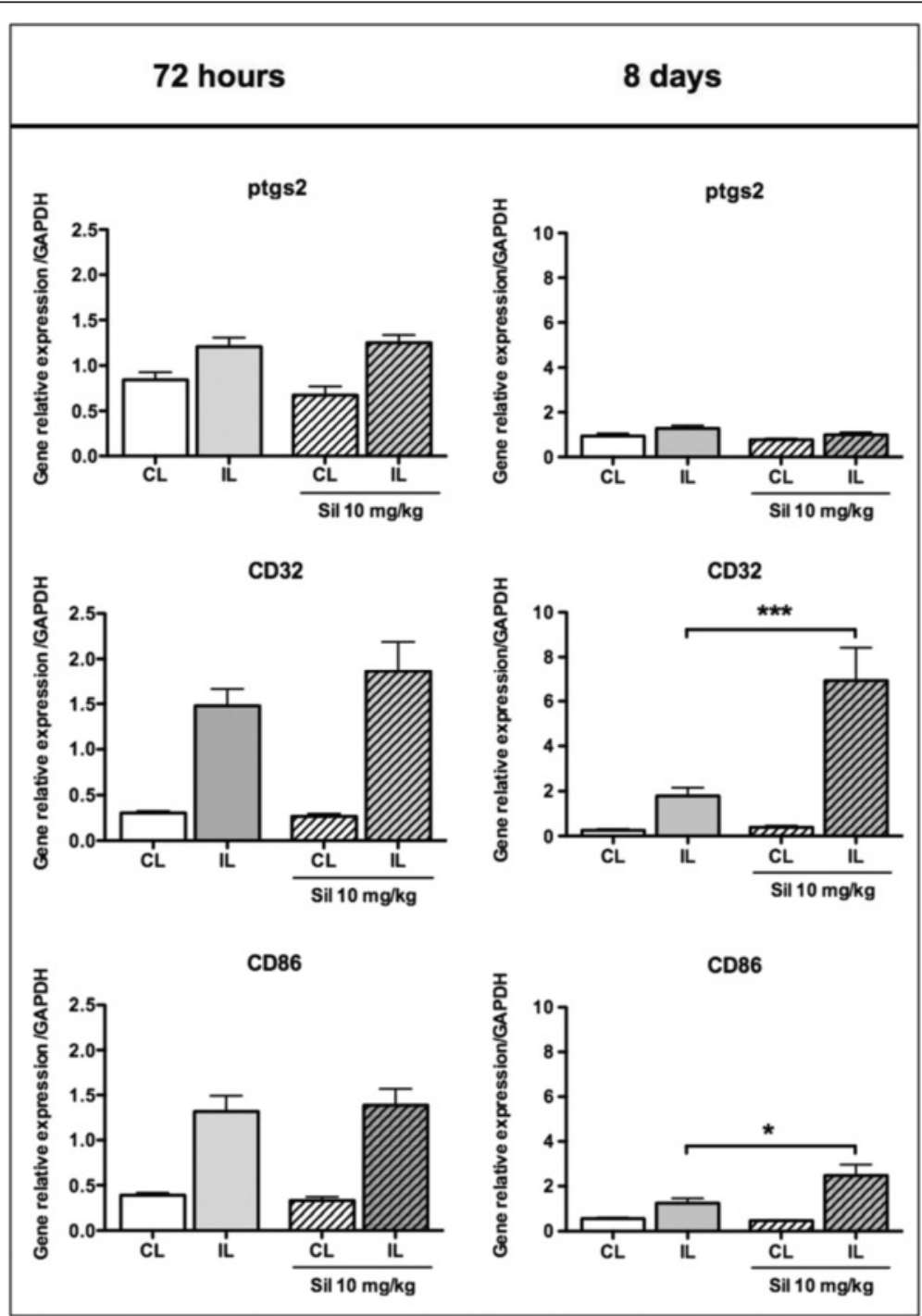

Fig. 4 Gene expression of microglia/macrophage M1-like markers in PBS- and sildenafil (Sil)-treated animals, $72 \mathrm{~h}$ and 8 days after pMCAo. M1 markers were measured in the ipsilateral (IL) and contralateral (CL) sides in PBS (plain bars) and sil-10 (hatched bars) animals. Data are mean \pm SEM. Data were assessed via an ANOVA, and when significant, the results of the Newman-Keuls post-test are shown; ${ }^{*} p<0.05$, and ${ }^{* * *} p<0.001$, compared to PBS 

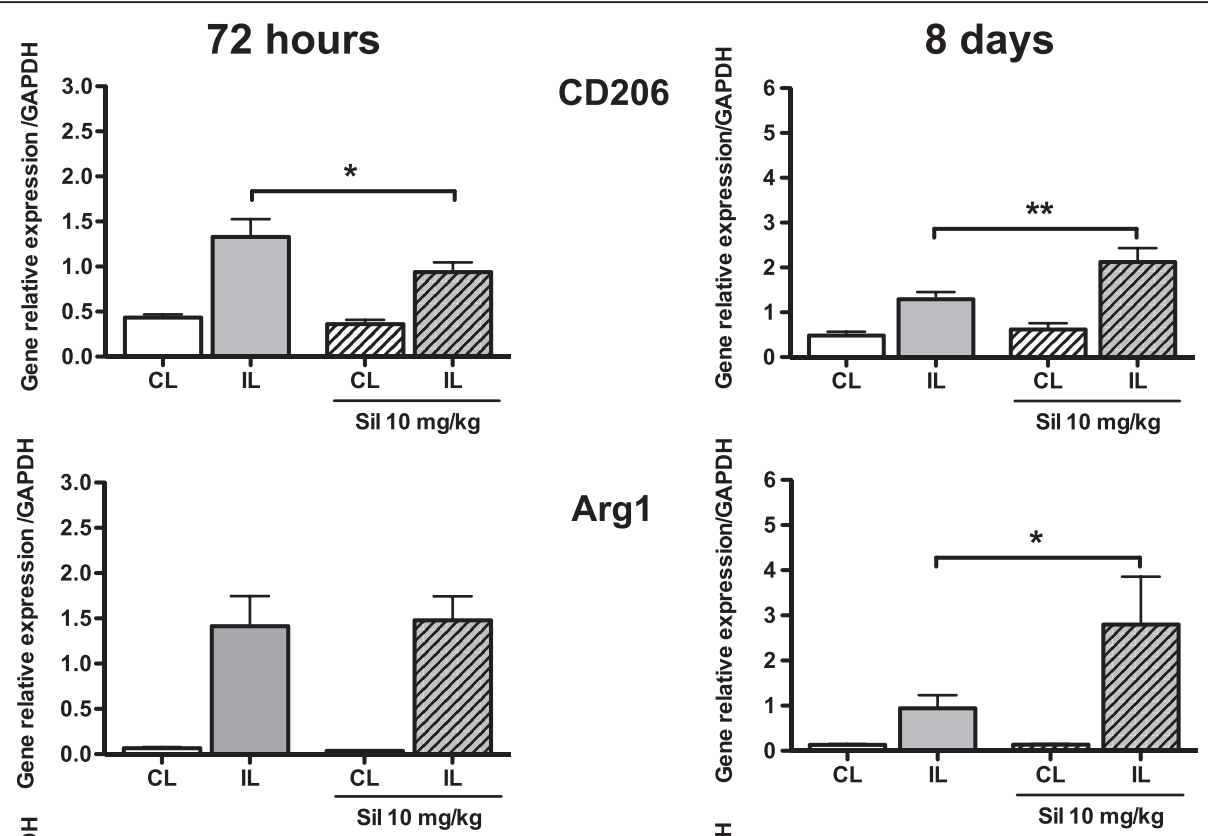

Arg1
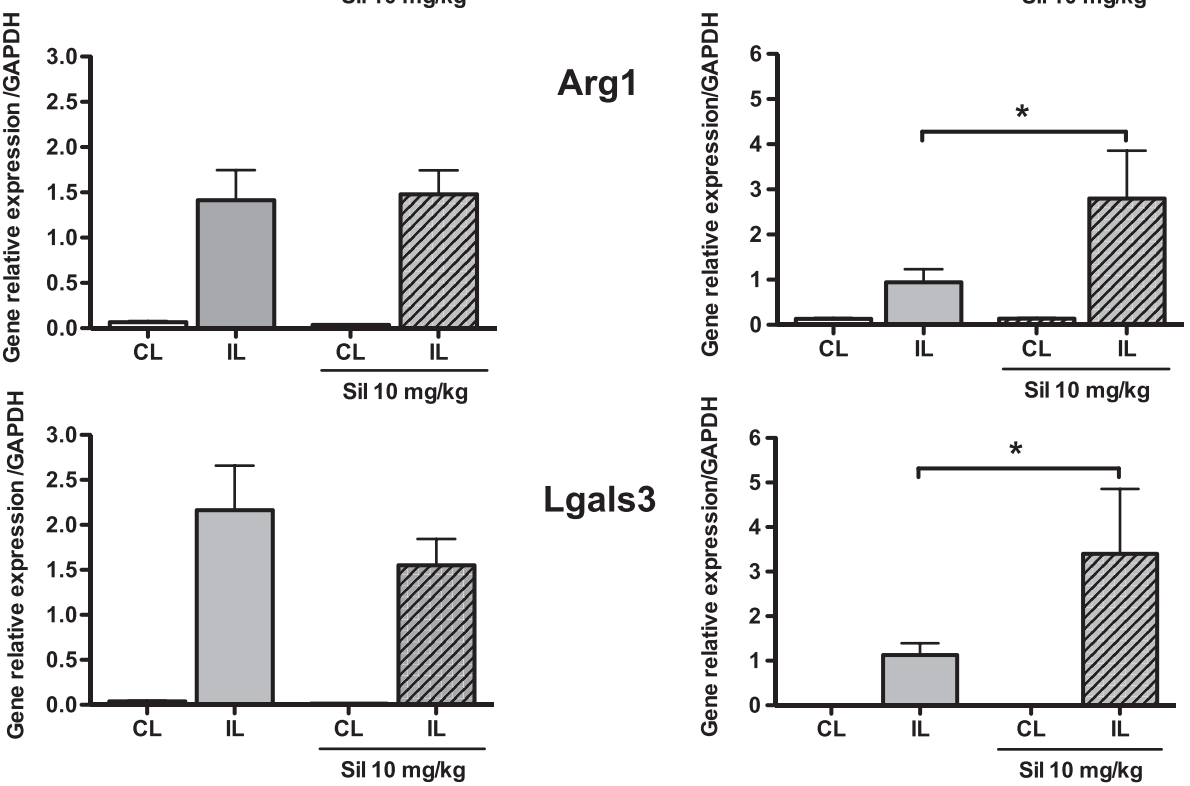

Lgals3
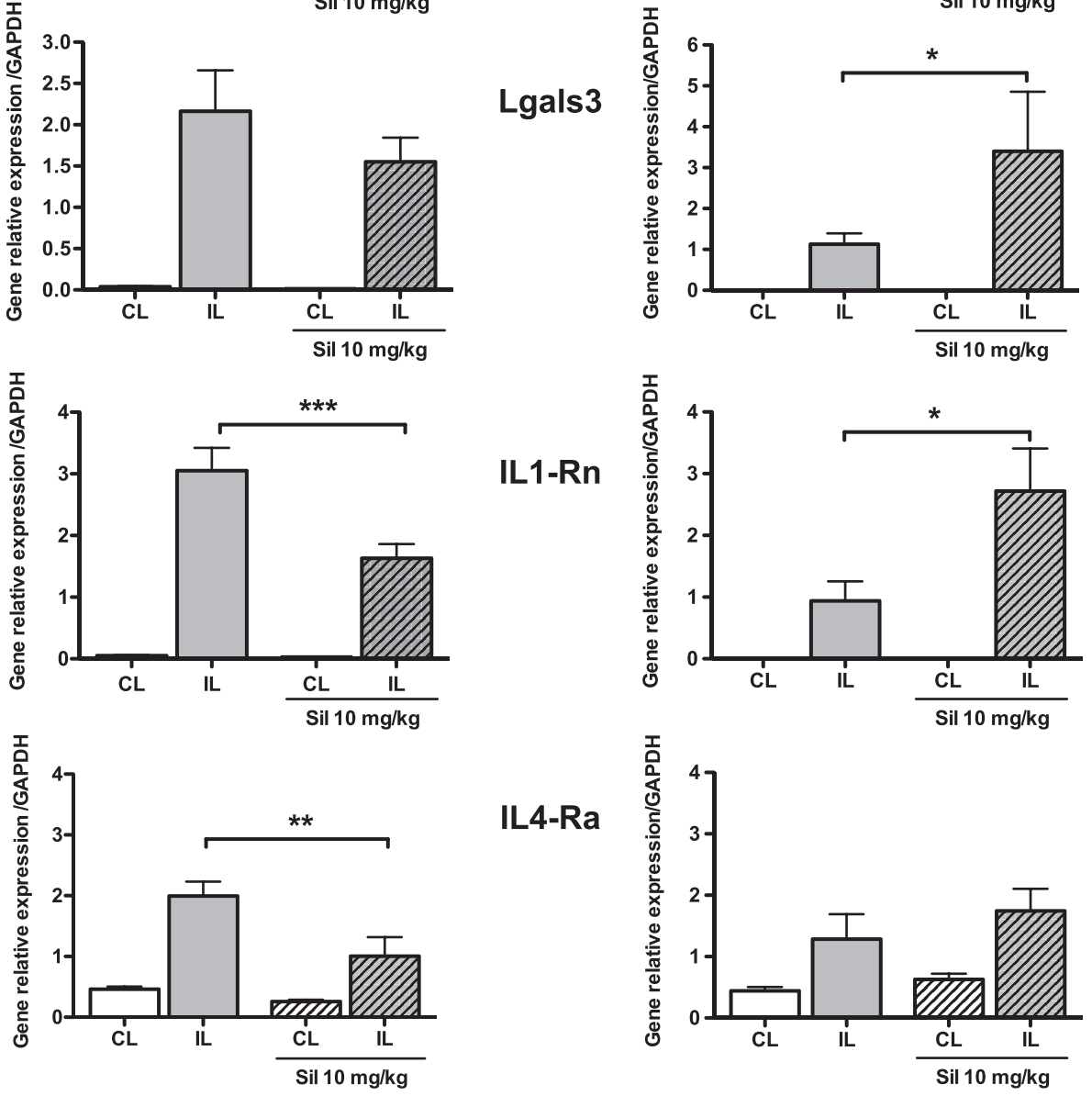

Fig. 5 (See legend on next page.) 
(See figure on previous page.)

Fig. 5 Gene expression of microglia/macrophage M2-like markers in PBS- and sildenafil (Sil)-treated animals, 72 h and 8 days after pMCAo. M2

markers were measured in the ipsilateral (IL) and contralateral (CL) side in PBS- (plain bars) and sil-10 (hatched bars) animals. Data are mean \pm SEM.

Data were assessed via an ANOVA, and when significant, the results of the Newman-Keuls post-test are shown; ${ }^{*} p<0.05,{ }^{* *} p<0.01$, and

${ }^{* * *} p<0.001$, compared to PBS

penumbra in $10 \mathrm{mg} / \mathrm{kg}$ (but not $0.5 \mathrm{mg} / \mathrm{kg}$ ) sildenafiltreated animals (Fig. 3b-d). In contrast, 8 days after pMCAo the number of microglia/macrophages stained by Iba- 1 were significantly reduced by sildenafil treatment $(0.5$ and/or $10 \mathrm{mg} / \mathrm{kg}$ dose) (Fig. 6b).

We then focused on the phenotype of microglial cells at both transcriptional (Figs. 4 and 5) and translational (Figs. 6 and 7) levels in the cortical tissue obtained from PBS- and sildenafil-treated animals. Seventy-two hours after pMCAo and PBS treatments, most of the markers (M1- and M2-like) were increased with the exception of ptgs2 (COX-2). As compared to PBS, sildenafil $(10 \mathrm{mg} / \mathrm{kg})$ did not change the expression of M1-like (ptgs2, CD32 and $C D 86$ ) markers $72 \mathrm{~h}$ after ischemia. In contrast, a significant increase was measured for $C D 32$ and $C D 868$ days after pMCAo (Fig. 4). At $72 \mathrm{~h}$ after pMCAo, sildenafil treatment $(10 \mathrm{mg} / \mathrm{kg})$ significantly decreased mRNA expression of M2-like markers (CD206, IL1-Rn, and IL4-Ra) but not Arg-1 and Lgals3. In contrast, most of these markers (CD206, Arg-1, Lgals3 and IL1-Rn) were increased 8 days after pMCAo (Fig. 5).

To further address the effect of sildenafil treatment on M1 and M2 markers, we examined the co-localization of Iba- $1^{+}$(a marker for both M1 and M2 microglia/macrophages) with either the M2-like marker Arg-1 (Fig. 6a, upper panel) or the M1-like marker COX-2 (Fig. 6a, lower panel) in the cortical penumbra. While a global decrease of Iba- $1^{+}$microglia/macrophage was observed by sildenafil irrespective of dosage (Fig. 6b), an increase of Iba- $1^{+} \mathrm{Arg}-1^{+}$double-positive M2 microglia/macrophage was observed at 10 but not $0.5 \mathrm{mg} / \mathrm{kg}$ (Fig. 6c), suggesting that a high dosage of sildenafil was required for microglia/ macrophages to obtain the M2 status. Interestingly, sildenafil decreased the number of Iba- $1^{+} \mathrm{COX}-2^{+}$double-positive M1 microglia/macrophages irrespective of dosage (Fig. 6d), suggesting it does not only affect phenotype polarization and that a lower dose threshold is required for inhibition of neurotoxic M1-type than for activation of neuroprotective M2-type microglia/ macrophages.

\section{Co-expression of M1- and M2-like markers in perivascular microglia/macrophages}

The macrophage mannose receptor MRC-1 (CD206), a marker for M2-type microglia/macrophages, was mainly detected in the leptomeninges from where they entered the neocortex along the penetrating arterioles in the penumbral tissue at $72 \mathrm{~h}$ (Fig. 7) and 8 days after
pMCAo (data not shown). On confocal images, we observed the co-localization of MRC-1 with the M1 marker COX-2 (Fig. 7a) indicating either a mixed phenotype or lack of specificity of this marker. MRC-1 expression was present in macrophages/microglia localized around microvessels labeled with the endothelial marker Glut-1 (stained with $54 \mathrm{kDa}$ Glut-1 protein, which regulates import of glucose from the blood to the brain across the endothelial cells of the blood-brain barrier) and present in the penumbra (Fig. 7b and Additional file 1: Figure S6). A few COX$2^{+} /$Arg- $1^{+}$-cells (2-4 cells per brain section) were still detected in the leptomeninges in both PBS- (Fig. 7c) and sildenafil- (10 mg/kg, data not shown) treated animals 8 days after pMCAo.

\section{Discussion}

In this study, we show that brain lesions induced by pMCAo in P9 mouse pups evolve between $72 \mathrm{~h}$ and 8 days post-ischemia and that the selective inhibition of PDE- 5 by sildenafil dose-dependently reduces lesion extent at 8 days post-pMCAo. The protective action of sildenafil appeared to involve a reduction in the overall number of microglia/macrophages in the late phase of lesion development as well as their polarization towards a neuroprotective M2 phenotype.

Whereas lesion volumes decrease after pMCAo in adult mice between $24 \mathrm{~h}$ and 7 days [14], they increased between 3 and 8 days in neonatal mice, as previously shown in neonatal rat [15]. Whether this difference reflects upon continued tissue destruction even in late phases after occlusion or reduced growth potential of the neonatal brain is yet unclear. Sildenafil treatment reduced lesion size at 8 days post-pMCAO, similar to that found at $72 \mathrm{~h}$, suggesting that sildenafil prevents lesion enlargement. We previously reported that sildenafil treatment, associated with an early significant increase in cerebral blood flow (CBF), reduces hypoxic-ischemic damage in the P7 rat brain [4]. By using both US and LSC imaging, we were here unable to observe early hemodynamic changes in either sildenafil- or PBStreated mice. A high variability in the number of leptomeningeal and/or pial vessels may explain the hemodynamic differences observed between the rat and mouse. Indeed, all ischemic models in the rat combine transient occlusion of two arteries at the same time, either pMCAo with transient occlusion of one or both common carotid arteries $[2,16]$ or blockade of the past external carotid artery-internal carotid artery bifurcation 


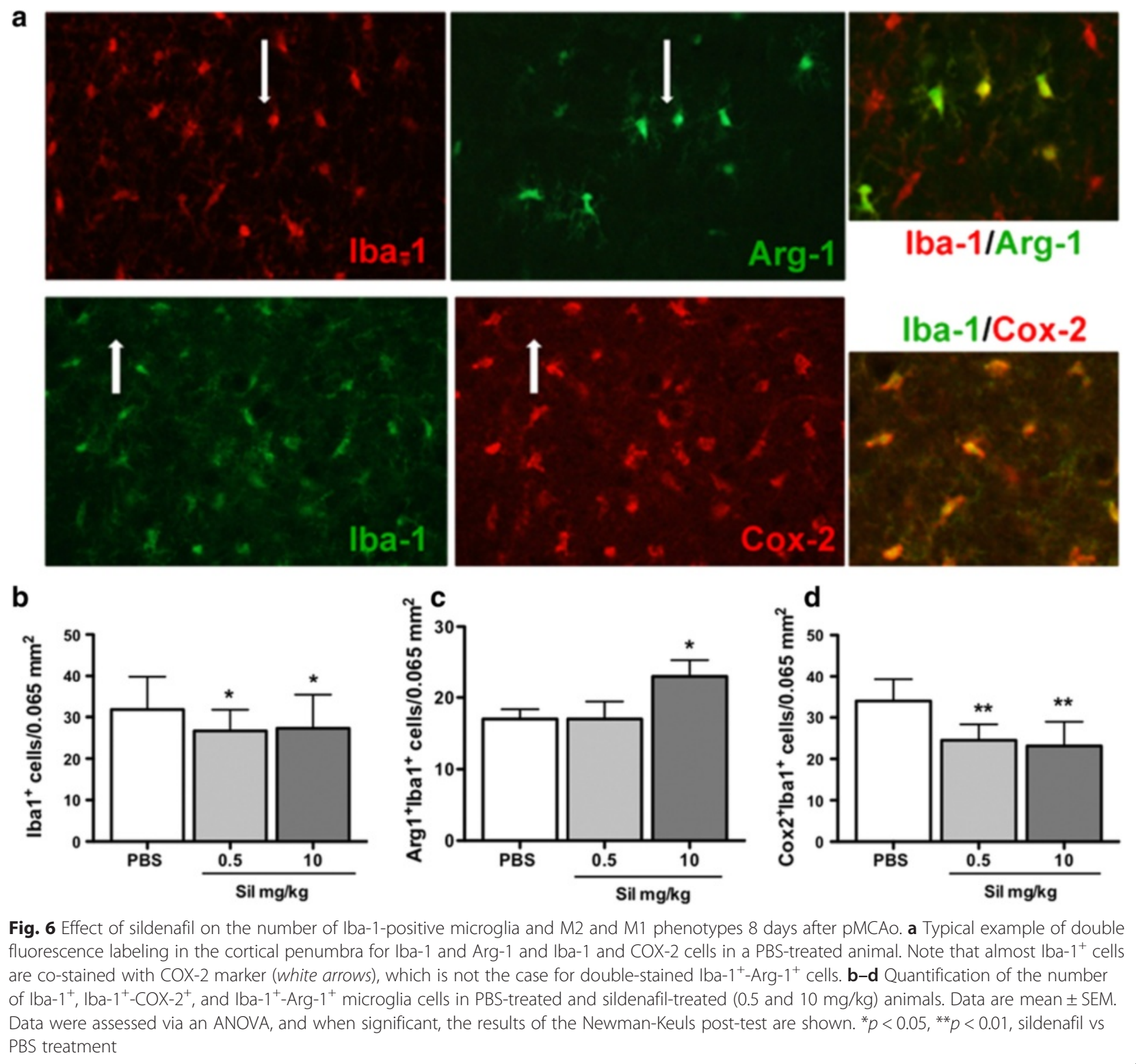

$[17,18]$. In contrast, a single pMCAo is sufficient to create ischemic lesions in mice [19]. Together, this suggests that combined occlusion of carotid and MC arteries is necessary to drop the BF in the ipsilateral hemisphere sufficiently for anastomoses to no longer be efficient despite the circle of Willis and to create a lesion in the rat. As BF was only measured up to $90 \mathrm{~min}$ after pMCAo, we cannot exclude the contribution of late collateral opening and patency. Together, our data suggest that sildenafil, although given shortly after pMCAo, may have other neuroprotective effects that may develop at later time points of recovery.

The impact of inflammation on post-injury outcome in the developing brain is increasingly recognized. In our pMCAo model in the P9 mouse, strong astrocytic and microglial responses were detected $72 \mathrm{~h}$ after injury and persisted for more than 1 week (personal data). We show that sildenafil potentiates reactive astrogliosis $72 \mathrm{~h}$ after pMCAo, in agreement with a previous report following focal cryolesions onto the cortex in adult rats treated with zaprinast and killed 3 days thereafter [9]. Zaprinast was expected to accelerate the formation of the glial scar and the regeneration of the injured tissue [10]. Sildenafil similarly increased GFAP immunoreactivity in adult mice submitted to a cortical cryolesions [20]. After brain injury, NO-dependent cGMP formation can occur in the astrocytes following NO synthase-2 induction in activated astrocytes and infiltrating macrophages $[21,22]$. Natriuretic peptides (NPs) comprising atrial (ANP), brain NP (BNP), and C-type (CNP) share the 


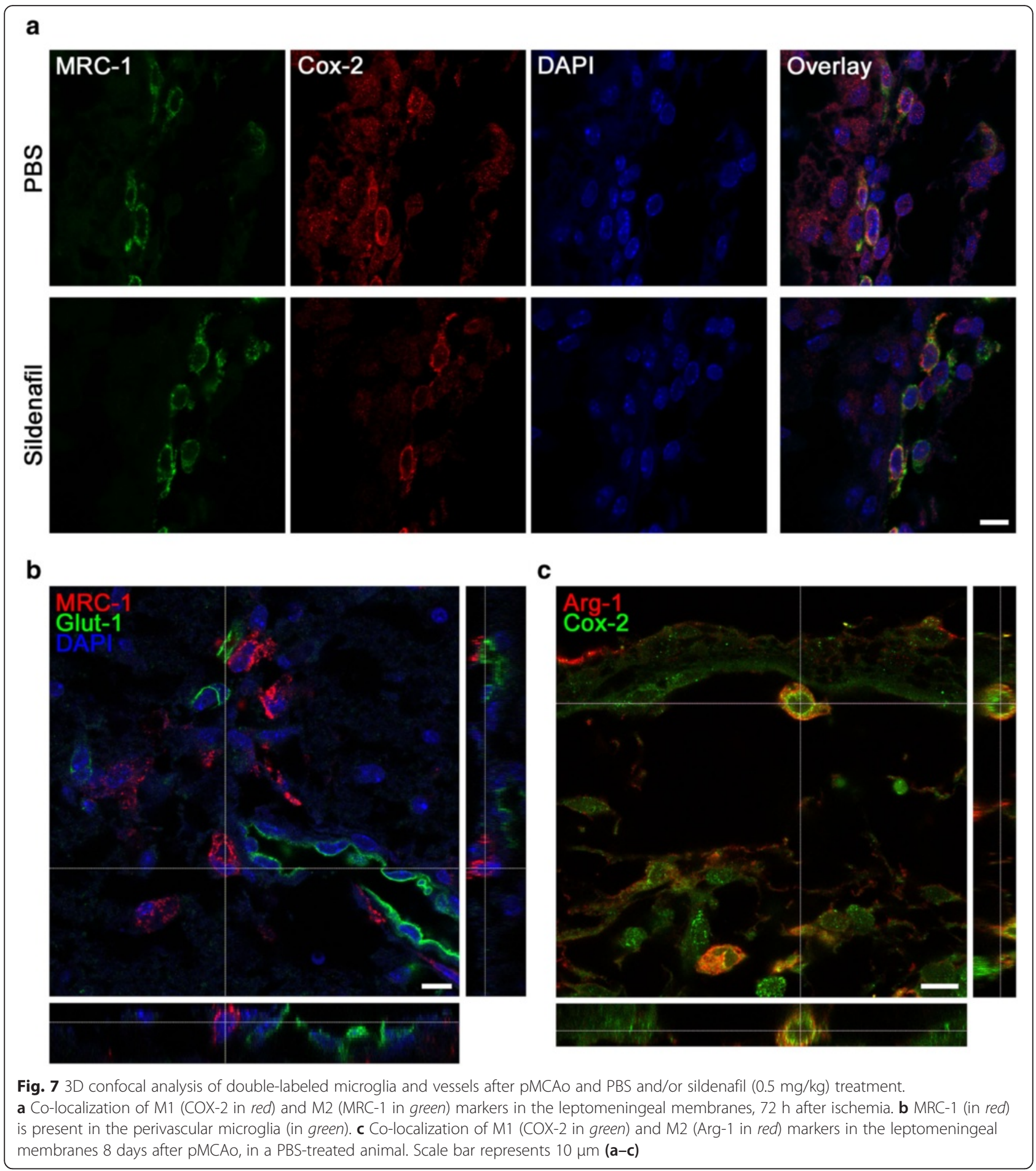

same intracellular signal transduction pathways with cGMP/cGMP-dependent protein kinase (cGK) as well as the NO pathway. The NPs/NO/cGMP/cGK pathway has been reported to increase $\mathrm{BF}$ and to be critical for neovascularization in vivo [23]. Interestingly, the content of ANP has been reported to increase in reactive astrocytes 3 days after injury in the white matter (WM) from autopsied human brain specimens after brain infarction and upregulation of the ANP/cGMP pathway (ANP is a vasorelaxant) may contribute to an increase in $\mathrm{BF}$ in response to the BF reduction in the infarcted area [24]. The increase of $\mathrm{GFAP}^{+}$cells observed here 3 days after pMCAo might be related to an increase in ANP and subsequent BF that we did not evaluate and could 
further explain the delayed reduction in astrogliosis observed at 8 days after pMCAo.

Another important finding of this study is that sildenafil reduces the recruitment and activation of microglia/ macrophages in the penumbral tissue at both $72 \mathrm{~h}$ and 8 days after pMCAo, as found for zaprinast in the cortical cryolesion model [9]. Microglial activation may be beneficial or harmful depending on polarization towards a pro-inflammatory $\mathrm{M} 1$ or an anti-inflammatory and pro-healing M2 phenotype [25]. Information regarding the expression of M1 and M2 markers and their temporal and spatial evolution in the ischemic brain are largely lacking. Changes in mRNA expression of M1 and M2 markers were maximally increased between 3-5 and 14. days after transient MCAo in the adult C57BL/6 mice [26].

In our neonatal mouse model, we observed an impact of sildenafil treatment on macrophage/microglia activation and the expression of M1- and M2-like markers. Whereas sildenafil only increased M1-like genes 8 days after ischemia, it decreased M2-like genes expression at the same time point. Cyclooxygenases (COX-1 and COX-2), which produce vasodilatory prostaglandins [27], contribute to neurovascular coupling [28] and could contribute to vasodilation in the penumbra and a subsequent absence of lesion extension in animals treated with sildenafil. Furthermore, cerebrovascular dilations to hypercapnia were demonstrated to be prostanoid dependent and nitric oxide independent in the newborn pig brain $[29,30]$. The combined increase of potential vasodilatory mediators in astrocytes and $\mathrm{COX}-2^{+}$cells in the penumbra at $72 \mathrm{~h}$ could, therefore, induce patency and/or recruitment of collaterals leading to a reduced damage 5 days after (at P17).

A significant upregulation of M2-like mRNA was detected after sildenafil treatment. In the adult rodent, M1 markers (iNOS, CD11b, CD16, and CD32) were still elevated at 14 days after stroke, while M2 markers (CD206, $\operatorname{Arg}-1, Y m 1 / 2$ and $I L-10)$ were decreased at 7 days after ischemia [31]. The M2 phenotype has a stronger capacity to elicit phagocytosis of dead neurons to avoid secondary inflammatory response and promote tissue regeneration, probably by the $\mathrm{ANP} / \mathrm{cGMP} / \mathrm{cKG}$ pathway [32]. We suggest a shift in polarization towards the M2 phenotype may contribute to the ability of sildenafil to prevent the late-stage extension of lesions in our model. We also found MRC-1 (CD-206) protein in infiltrating macrophages (in the leptomeninges), as previously reported after hypoxia-ischemia in the P7 rat [33]; however, we further demonstrate that $\mathrm{MRC}-1^{+} / \mathrm{CD} 206$ perivascular microglia/macrophages entered the brain via penetrating microvessels. In contrast $\mathrm{CD}-206^{+}$cells were exclusively located in the ischemic core in adult mice $24 \mathrm{~h}$ after pMCAo [34]. Interestingly, mixed transitional (Mtran) phenotype has been introduced to characterize double-labeled M1-M2 macrophage/microglia following traumatic brain injury [35]. Such $\mathrm{MRC}-1^{+} / \mathrm{COX}-$ $2^{+}$and Arg- $1^{+} / \mathrm{COX}-2^{+}$macrophage/microglia in the leptomeninges may also represent a transitional Mtran phenotype in our ischemic model.

\section{Conclusions}

In conclusion, our study suggests that sildenafil may prevent the progression of the ischemic lesions in neonatal mice through the modulation of inflammatory pathways including microglial/macrophage polarization. Further investigation is needed to elucidate mechanisms that govern the change in microglia/macrophage phenotype and the regulation of lesion size in the neonatal rodent brain. As the use of sildenafil is indicated for idiopathic pulmonary hypertension [36], it could be explored for immunomodulatory stroke treatment in children.

\section{Additional files}

Additional file 1: Figures S1-S6. Figure S1. Outline of the experimental procedure in P9 C57Bl/6 mice subjected to pMCAo. Figure S2. A: mean blood flow velocities (BFVs) in the left and right ICA (IICA, rICA) and basilar trunk (BT) in basal conditions and 15 min after pMCAo. *, \#p<0.05 vs basal for each artery. B Variation in arterial blood flow (BF) in P9 mice subjected to PMCAo and administered with PBS (white bars) and/or sildenafil (15 mg/kg, black bars). Figure S3. cGMP dosage in the ipsilateral hemisphere in the control (PBS-treated naive mice, $n=5)$ and in sildenafil- $(10 \mathrm{mg} / \mathrm{kg})$ treated mice, at 1 and $3 \mathrm{~h}(\mathrm{~h})$ after treatment. ${ }^{* * *} p<0.001$ vs PBS. Figure S4. Quantification of GFAP-positive cells in the penumbra at $72 \mathrm{~h}$ and 8 days after pMCAo in PBS- and sildenafiltreated animals ( $n=8$ per group). Figure S5. Immunohistochemistry for astrocyte (GFAP; A, B) and macrophage (tomato lectin (TL) C-D)/microglia (Iba-1; E, F) in PBS-treated animals (A, C, and E) and sildenafil $10 \mathrm{mg} / \mathrm{kg}$ $(B, D$, and $F)$ at $72 h(A-D)$ and 8 days $(E, F)$ after pMCAo in mouse pups. Figure S6. 3D confocal reconstruction of a microvessel stained with the 54 kDa Glut-1 protein (green) and microglia/macrophage stained with MRC-1 protein (M2-like marker). (PDF $1359 \mathrm{~kb}$ )

Additional file 2: Table S1. Primer sequences, protein targets, and $\mathrm{NCBI}$ references. (PDF $306 \mathrm{~kb}$ )

\section{Competing interests}

The authors declare that they have no competing interests.

\section{Authors' contributions}

$\mathrm{RM}, \mathrm{CCM}$, and $\mathrm{OB}$ conceived and designed the study. RM, PLL, and VB conducted the experimental protocols. RM, LD, AG, and JP performed the histology, histochemistry, and qPCR. ZC performed the confocal analysis. RM, $L D$, and $P B$ performed the ultrasound imaging. $R M$ and PLL performed the laser speckle contrast imaging. RM, PLL, VB, PB, OB, and CCM analyzed and interpreted data. CCM, RM, and $\mathrm{OB}$ wrote the manuscript with input from all authors. All authors read and approved the final manuscript.

\section{Acknowledgements}

All the authors are indebted to Dr. E. Camerer (INSERM U970) for critical revision and editing this manuscript.

\section{Author details}

'University Paris Diderot, Sorbonne Paris Cité, INSERM, UMR 1141, 75019 Paris, France. ${ }^{2}$ University degli Studi di Udine, Udine, Italy. ${ }^{3}$ UPMC-Paris6, AP-HP, Hôpital Armand Trousseau, Réanimation Néonatale et Pédiatrique, 75012 Paris, France. ${ }^{4}$ Pharmacologie de la Circulation Cérébrale - EA4475, 
Faculté des Sciences Pharmaceutiques et Biologiques, University of Paris Descartes, Paris, France. ${ }^{5}$ University Paris Diderot, Sorbonne Paris Cité, AP-HP, Hôpital Lariboisière, Physiologie Clinique, Explorations-Fonctionnelles, 75010 Paris, France. 'University Paris Diderot, Sorbonne Paris Cité, INSERM, U965, 75010 Paris, France. ${ }^{7}$ University Paris Diderot, Sorbonne Paris Cité, AP-HP, Hôpital Robert Debré, Urgences Pédiatriques, 75019 Paris, France. ${ }^{8}$ University Paris Diderot, Sorbonne Paris Cité, AP-HP, Hôpital Robert Debré, Réanimation Néonatale, 75019 Paris, France. ${ }^{9}$ INSERM UMR 1141, Hopital Robert Debré, 48 bd Serurier, 75019 Paris, France.

\section{Received: 28 December 2015 Accepted: 24 April 2016}

\section{Published online: 28 April 2016}

\section{References}

1. Chabrier S, Husson B, Dinomais M, Landrieu P, Nguyen The Tich S. New insights (and new interrogations) in perinatal arterial ischemic stroke. Thromb Res. 2011;127:13-22.

2. Charriaut-Marlangue $\mathrm{C}$, Bonnin P, Leger PL, Renolleau S. Brief update on hemodynamic responses in animal models of neonatal stroke and hypoxiaischemia. Exp Neurol. 2013;248:316-20.

3. Wallis RM, Corbin JD, Francis SH, Ellis P. Tissue distribution of phosphodiesterase families and the effects of sildenafil on tissue cyclic nucleotides, platelet function, and the contractile responses of trabeculae carneae and aortic rings in vitro. Am J Cardiol. 1999;83:3C-12.

4. Charriaut-Marlangue C, Nguyen T, Bonnin P, Duy AP, Leger PL, Csaba Z, et al. Sildenafil mediates blood-flow redistribution and neuroprotection after neonatal hypoxia-ischemia. Stroke. 2014;45:850-6.

5. Bednar MM. The role of sildenafil in the treatment of stroke. Curr Opin Investig Drugs. 2008;9:754-9.

6. Nunes AK, Raposo C, Luna RL, Cruz-Hofling MA, Peixoto CA. Sildenafil (Viagra(R)) down regulates cytokines and prevents demyelination in a cuprizone-induced MS mouse model. Cytokine. 2012;60:540-51.

7. ladecola C, Anrather J. The immunology of stroke: from mechanisms to translation. Nat Med. 2011;17:796-808.

8. Murray PJ, Allen JE, Biswas SK, Fisher EA, Gilroy DW, Goerdt S, et al. Macrophage activation and polarization: nomenclature and experimental guidelines. Immunity. 2014;41:14-20.

9. Taylor RA, Sansing LH. Microglial responses after ischemic stroke and intracerebral hemorrhage. Clin Dev Immunol. 2013;2013:746068.

10. Pifarre P, Prado J, Giralt M, Molinero A, Hidalgo J, Garcia A. Cyclic GMP phosphodiesterase inhibition alters the glial inflammatory response, reduces oxidative stress and cell death and increases angiogenesis following focal brain injury. J Neurochem. 2010;112:807-17.

11. Charriaut-Marlangue C, Bonnin P, Gharib A, Leger PL, Villapol S, Pocard M, et al. Inhaled nitric oxide reduces brain damage by collateral recruitment in a neonatal stroke model. Stroke. 2012;43:3078-84.

12. Leger PL, Bonnin P, Moretti R, Tanaka S, Duranteau J, Renolleau S, et al. Early recruitment of cerebral microcirculation by neuronal nitric oxide synthase inhibition in a juvenile ischemic rat model. Cerebrovasc Dis. 2015:41:40-9.

13. Chhor V, Le Charpentier T, Lebon S, Ore MV, Celador IL, Josserand J, et al. Characterization of phenotype markers and neuronotoxic potential of polarised primary microglia in vitro. Brain Behav Immun. 2013;32:70-85.

14. Poittevin M, Bonnin P, Pimpie C, Riviere L, Sebrie C, Dohan A, et al. Diabetic microangiopathy: impact of impaired cerebral vasoreactivity and delayed angiogenesis after permanent middle cerebral artery occlusion on stroke damage and cerebral repair in mice. Diabetes. 2015;64:999-1010.

15. Renolleau S, Aggoun-Zouaoui D, Ben-Ari Y, Charriaut-Marlangue C. A model of transient unilateral focal ischemia with reperfusion in the P7 neonatal rat: morphological changes indicative of apoptosis. Stroke. 1998;29:1454-60. discussion 61.

16. Bonnin P, Leger PL, Deroide N, Fau S, Baud O, Pocard M, et al. Impact of intracranial blood-flow redistribution on stroke size during ischemiareperfusion in 7-day-old rats. J Neurosci Methods. 2011;198:103-9.

17. Ashwal S, Cole DJ, Osborne S, Osborne TN, Pearce WJ. L-NAME reduces infarct volume in a filament model of transient middle cerebral artery occlusion in the rat pup. Pediatr Res. 1995;38:652-6.

18. Derugin N, Ferriero DM, Vexler ZS. Neonatal reversible focal cerebral ischemia: a new model. Neurosci Res. 1998;32:349-53.

19. Tsuji M, Ohshima M, Taguchi A, Kasahara Y, Ikeda T, Matsuyama T. A novel reproducible model of neonatal stroke in mice: comparison with a hypoxiaischemia model. Exp Neurol. 2013;247:218-25.
20. Prado J, Pifarre P, Giralt M, Hidalgo J, Garcia A. Metallothioneins I/II are involved in the neuroprotective effect of sildenafil in focal brain injury. Neurochem Int. 2013;62:70-8.

21. Brahmachari S, Fung YK, Pahan K. Induction of glial fibrillary acidic protein expression in astrocytes by nitric oxide. J Neurosci. 2006;26:4930-9.

22. Murphy S. Production of nitric oxide by glial cells: regulation and potential roles in the CNS. Glia. 2000;29:1-13.

23. Yamahara $\mathrm{K}$, Itoh H, Chun TH, Ogawa Y, Yamashita J, Sawada N, et al. Significance and therapeutic potential of the natriuretic peptides/cGMP/ CGMP-dependent protein kinase pathway in vascular regeneration. Proc Natl Acad Sci U S A. 2003;100:3404-9.

24. Nogami M, Shiga J, Takatsu A, Endo N, Ishiyama I. Immunohistochemistry of atrial natriuretic peptide in brain infarction. Histochem J. 2001;33:87-90.

25. Fumagalli S, Perego C, Pischiutta F, Zanier ER, De Simoni MG. The ischemic environment drives microglia and macrophage function. Front Neurol. 2015;6:81.

26. Hu X, Li P, Guo Y, Wang H, Leak RK, Chen S, et al. Microglia/macrophage polarization dynamics reveal novel mechanism of injury expansion after focal cerebral ischemia. Stroke. 2012;43:3063-70.

27. Funk CD. Prostaglandins and leukotrienes: advances in eicosanoid biology. Science. 2001;294:1871-5.

28. Stefanovic B, Bosetti F, Silva AC. Modulatory role of cyclooxygenase-2 in cerebrovascular coupling. Neuroimage. 2006;32:23-32.

29. Leffler CW, Busija DW. Prostanoids and pial arteriolar diameter in hypotensive newborn pigs. Am J Physiol. 1987;252:H687-91.

30. Willis AP, Leffler CW. NO and prostanoids: age dependence of hypercapniaand histamine-induced dilations of pig pial arterioles. Am 」 Physiol. 1999;277:H299-307.

31. Xia CY, Zhang S, Gao Y, Wang ZZ, Chen NH. Selective modulation of microglia polarization to M2 phenotype for stroke treatment. Int Immunopharmacol. 2015;25:377-82

32. Miro-Mur F, Perez-de-Puig I, Ferrer-Ferrer M, Urra X, Justicia C, Chamorro A, et al. Immature monocytes recruited to the ischemic mouse brain differentiate into macrophages with features of alternative activation. Brain Behav Immun. 2015. doi:10.1016/j.bbi.2015.08.010.

33. Bonestroo HJ, Nijboer $\mathrm{CH}$, van Velthoven $\mathrm{CT}$, Kavelaars A, Hack $\mathrm{CE}$, van Bel F, et al. Cerebral and hepatic inflammatory response after neonatal hypoxiaischemia in newborn rats. Dev Neurosci. 2013;35:197-211.

34. Perego C, Fumagalli S, De Simoni MG. Temporal pattern of expression and colocalization of microglia/macrophage phenotype markers following brain ischemic injury in mice. J Neuroinflammation. 2011;8:174.

35. Kumar A, Alvarez-Croda DM, Stoica BA, Faden Al, Loane DJ. Microglia/ macrophage polarization dynamics following traumatic brain injury. J. Neurotrauma, 2016, in press. PMID: 26486881.

36. Fraisse $\mathrm{A}$, Wessel $\mathrm{DL}$. Acute pulmonary hypertension in infants and children: cGMP-related drugs. Pediatr Crit Care Med. 2010;11:S37-40.

\section{Submit your next manuscript to BioMed Central and we will help you at every step:}

- We accept pre-submission inquiries

- Our selector tool helps you to find the most relevant journal

- We provide round the clock customer support

- Convenient online submission

- Thorough peer review

- Inclusion in PubMed and all major indexing services

- Maximum visibility for your research

Submit your manuscript at www.biomedcentral.com/submit
Biomed Central 